OPEN ACCESS

Edited by:

Gang Wu,

VU University Amsterdam,

Netherlands

Reviewed by:

Janos Kanczler,

University of Southampton,

United Kingdom

Dongxia Ye,

University of Rochester, United States

*Correspondence:

Guoyu Lv

lgy929@126.com

${ }^{+}$These authors have contributed equally to this work

Specialty section:

This article was submitted

to Biomaterials,

a section of the journal

Frontiers in Materials

Received: 23 August 2020

Accepted: 30 October 2020

Published: 11 December 2020

Citation:

Peng H, Li J, Xu Y and Lv G (2020)

Icaritin Enhancing Bone Formation

Initiated by Sub-Microstructured

Calcium Phosphate Ceramic for

Critical Size Defect Repair.

Front. Mater. 7:598057.

doi: 10.3389/fmats.2020.598057

\section{Icaritin Enhancing Bone Formation Initiated by Sub-Microstructured Calcium Phosphate Ceramic for Critical Size Defect Repair}

\author{
Haitao Peng ${ }^{1 \dagger}$, Jianxiao $\mathrm{Li}^{2 \dagger}$, Yanan $\mathrm{Xu}^{3}$ and Guoyu $\mathrm{Lv}^{1 *}$ \\ ${ }^{1}$ College of Physics, Sichuan University, Chengdu, China, ${ }^{2}$ Department of Orthopedics, The First Affiliated Hospital of Chongqing \\ Medical University, Chongqing, China, ${ }^{3}$ Department of Trauma Surgery, Emergency Medical Center of Chongqing, Chongqing, \\ China
}

Adequate bone tissue regeneration has been challenging to achieve at critical-sized bone defects caused by disease. Bone tissue engineering using a combination of scaffolds and bioactive factors provides new hope for the treatment of this extreme condition. Icaritin, a herb-derived chemical, has shown its ability to enhance bone formation both in vitro and in vivo, and it has been found that sub-micron surface structure instructs bone formation in calcium phosphate ceramics (CaPs). Here, we evaluated the possibility of using a submicron surface structured $\mathrm{CaP}$ ceramic as the carrier of icaritin for bone tissue regeneration in critical-sized bone defects. Icaritin, an herb-derived chemical, was loaded into a submicron surface structured porous calcium phosphate ceramic $(\varnothing 12.8 \times 3 \mathrm{~mm})$ to get samples with $0,10,50,250$, and 1,250 $\mu \mathrm{g}$ icaritin per $\mathrm{CaP}$ disc (M0, M10, M50, M250, M1250 groups, respectively). In vitro evaluation with the certain dosages correlated to those released from the samples showed a dose-dependent enhancement of osteogenic differentiation and mineralization of human bone marrow stromal cells with the presence of osteogenic factors in the culture medium, indicating icaritin is an osteopromotive factor. After intramuscular implantation of the samples in dogs for 8 weeks, a dose-dependent of bone formation was seen with enhanced bone formation at the dosage of 50 and $250 \mu \mathrm{g}$. To evaluate the in vivo osteogenic potentials of icaritin-containing $\mathrm{CaP}$ ceramic scaffolds in the orthopedic site, a $12.8 \mathrm{~mm}$ calvarial defect model in rabbits was established. Micro-computed tomography (micro-CT) and histology results at weeks 4, 8 and 12 post-surgery showed more newly formed bone in M250 group, with correspondingly more new vessel ingrowth. The results presented herein suggested that being osteopromotive, icaritin could enhance bone formation initiated by sub-microstructured CaP ceramics and the CaP ceramics scaffold incorporating icaritin is a promising biomaterial for the treatment of critical-sized defect.

Keywords: icaritin, submicron surface structure, calcium phosphate ceramic, critical-sized bone defect, bone regeneration 


\section{INTRODUCTION}

The orthopedic critical-sized defect due to traumatic injuries or tumor resection is still a significant challenge. Pursuing alternatives to autologous bone grafts has always been a goal of biomaterial engineering. Allowing bone formation on their surface and form chemical bonding to the new bone, calcium phosphate $(\mathrm{CaP})$ ceramics have been widely used as bone graft substitutes (Hench and Wilson, 1984; Damien and Parsons, 1991; Bohner et al., 2012). However, compared with autogenous bone, the capacity of $\mathrm{CaP}$ ceramics for repairing critical-sized defects still seems to be insufficient.

Surface modification may improve the bone regeneration ability of $\mathrm{CaP}$ ceramics. When the surface structure of $\mathrm{CaP}$ ceramics decreased from micron to submicron scale, it induced bone formation in the muscle of dogs (Davison et al., 2014b; Zhang et al., 2014)and under the skin of FVB mouse (Davison et al., 2014a). Having the osteoinductive property, calcium phosphate ceramics not only enhanced bone regeneration in non-critically sized bone defects (Habibovic et al., 2004; Yuan et al., 2006) but also could repair criticalsized defects (Yuan et al., 2010) and enhance bone formation in the spinal environment (van Dijk et al., 2018).

Besides, osteogenic factors can also exert considerable bone repair effects when combined with scaffolds (McMillan et al., 2018). Icaritin, an exogenous semi-synthesized small phytomolecule, is an intestinal metabolite of herbal epimedium-derived flavonoids (Zhang et al., 2007; Qin et al., 2008), and has shown to affect various biological processes. For instance, it inhibited both thrombosis and lipid-deposition reducing thus the incidence of steroid-associated osteonecrosis (Zhang et al., 2009), and it exhibited in vitro anti-inflammatory effects on the mouse peritoneal macrophages (Lai et al., 2013). Most recently, the roles of icaritin in bone metabolism were also found. It enhanced the differentiation and proliferation of osteoblasts, facilitated matrix calcification (Qin et al., 2008; Yao et al., 2012), and enhanced bone regeneration in an ovariectomized rat osseous model (Zhang et al., 2008; Peng et al., 2013).

Although icaritin exhibits bone-enhancing effects in PLGA/ TCP scaffolds (Chen et al., 2012; Chen et al., 2013), there is no report on the combination of icaritin and inorganic ceramics to repair the critical-sized defect. More notably, when icaritin was loaded on a special submicron structured $\mathrm{CaP}$ scaffold, it is unknown whether the bone regeneration ability of $\mathrm{CaP}$ ceramics is enhanced.

We hypothesized that the combination of chemicals enhancing bone formation and osteoinductive calcium phosphate ceramics would result in bone substitutes with improved bone regeneration capacity for critical-sized defect repair. Considering the possible bone-enhancing function of icaritin as previously shown, we introduced icaritin into submicron surface structured tricalcium phosphate ceramic and characterized the resulting materials concerning for their physicochemical properties and icaritin release. Following the in vitro evaluation of icaritin released with hBMSCs, we investigated the bone-forming ability of the materials after intramuscular implantation in a canine model. According to previous research, rabbit calvarial defect is a mature model for evaluating the bone repairability of biological scaffolds, but the critical defect size rarely exceeds $10 \mathrm{~mm}$ (Xu et al., 2008; Lee et al., 2018). In this study, we designed a $12.8 \mathrm{~mm}$ critical bone defect to investigate the bone repair effect of the materials by microcomputed tomography (micro-CT) and histology. With results showing, the sub-microstructured $\mathrm{CaP}$ ceramics scaffold incorporating icaritin could be a promising biomaterial for the treatment of critical-sized defect.

\section{MATERIALS AND METHODS}

\section{Preparation and Physico-chemical Properties of CaP Ceramics With Sub-micron Scale Surface Structure}

$\mathrm{CaP}$ powder was synthesized by neutralization reaction method at $\mathrm{Ca} / \mathrm{P}$ ratio of $1.5 . \mathrm{H}_{3} \mathrm{PO}_{4}$ solution was dropped into a $\mathrm{Ca}(\mathrm{OH})_{2}$ suspension to get $\mathrm{CaP}$ powder. The porous $\mathrm{CaP}$ green bodies were obtained using $\mathrm{H}_{2} \mathrm{O}_{2}(1 \%)$ and wax particles and then sintered at $1,050^{\circ} \mathrm{C}$ for $8 \mathrm{~h}$ to form ceramics (Yuan et al., 2010; Zhang et al., 2014; Zhang et al., 2015). Microporous discs $(\varnothing 12.8 \times 3 \mathrm{~mm})$ were machined from the ceramic bodies using a lathe and a diamond saw microtome (Leica SP1600). Discs were ultrasonically cleaned in successive baths of acetone, ethanol, and deionized water, and then dried at $60^{\circ} \mathrm{C}$.

$\mathrm{X}$-ray diffraction (XRD; Rigaku, Tokyo, Japan) was used to analyze the crystal structure of $\mathrm{CaP}$ powder. The grain, micropore size and surface topography was detected by scanning electron microscope (XL30, ESEMFEG, Philips, Eindhoven, The The Netherlands). Especially, the grain and micropore was selected randomly from the image and the vertical lengths crossing the center of each grain and micropore were considered to be the grain and pore size. Mercury intrusion was used to obtain the ceramics' total porosity, microporosity and micropore size distribution.

\section{In vitro Release of Icaritin by CaP Scaffold in Calcium Medium}

$\mathrm{CaP}$ discs were sterilized with autoclave at $121^{\circ} \mathrm{C}$ for $30 \mathrm{~min}$. Icaritin solutions of $0,40,200,1$ and $5 \mathrm{mg} / \mathrm{ml}$ were prepared with methyl alcohol and sterilized with $0.2 \mu \mathrm{m}$ filter (Acrodisc ${ }^{\circledR} 13 \mathrm{CR}$ PTFE $0.2 \mu \mathrm{m}$, Gelman Pall). Under sterile condition, $250 \mu \mathrm{L}$ of each icaritin solution was loaded onto $\mathrm{CaP}$ disc. Thereafter, methyl alcohol was evaporated in sterile conditions at $50^{\circ} \mathrm{C}$ with ventilation for seven days to obtain samples containing $0 \mu \mathrm{g}$ icaritin per disc (M0), $10 \mu \mathrm{g}$ icaritin per disc (M10), $50 \mu \mathrm{g}$ icaritin per disc (M50), $250 \mu \mathrm{g}$ icaritin per disc (M250) and $1,250 \mu \mathrm{g}$ icaritin per disc (M1250) respectively. Then, samples $(n=3)$ were individually incubated in $3 \mathrm{ml}$ of culture medium containing $a$-MEM (Lonza), 10\% FBS (Gibco) and 1\% antibiotics (100 U/ml penicillin and $100 \mu \mathrm{g} / \mathrm{ml}$ streptomycin, Invitrogen) at $37^{\circ} \mathrm{C}$ and $5 \% \mathrm{CO}_{2}$. Culture medium was refreshed at day $1,2,4,7$, $14,21,28,35,42,49$ and day 56 respectively. The medium 
collected at each refreshment point were stored at $-20^{\circ} \mathrm{C}$ before further use.

Icaritin concentration in medium was detected with an HPLCMS/MS system (TSQ Quantum Ultra, ThermoFisher Scientific, Co. Ltd., USA) equipped with a surveyor Autosampler, a mass pump and a triple quadrupole mass spectrometer. Six standard icaritin solutions in culture medium (i.e., 2, 5, 50, 100, 250, and $500 \mathrm{ng} / \mathrm{ml}$ ) were prepared for a calibration line. The icaritin concentration in the samples was calculated according to the calibration line, while the remaining icaritin was calculated by subtracting the cumulative icaritin release from the original loading.

To determine the solubility of icaritin in water and culture medium, icaritin was directly added to water and culture medium at $37^{\circ} \mathrm{C}$ overnight and centrifuged at $5,000 \mathrm{rpm}$ for $5 \mathrm{~min}$. The supernatants $(n=3)$ were subjected to HPLC-MS/MS assay.

\section{In vitro Evaluation With Human Bone Marrow Stromal cells (hBMSC) \\ Medium Preparation}

Basic medium (BM), Growth medium (GM) and Osteogenic medium (OM) were used in the in vitro cell culture. Basic medium was $a$-MEM (Invitrogen, United Kingdom) supplemented with 10\% FBS (Lonza, Germany), $2 \mathrm{mM} \mathrm{L-}$ Glutamine (Invitrogen, United Kingdom), $0.2 \mathrm{mML}$-Ascorbic acid 2-phosphate (AsAP, Sigma), $100 \mathrm{IU} / \mathrm{ml}$ penicillin (Invitrogen, United Kingdom) and $100 \mathrm{~g} / \mathrm{ml}$ streptomycin (Invitrogen, United Kingdom), growth medium was basic medium supplemented with $1 \mathrm{ng} / \mathrm{ml}$ basic fibroblasts growth factors (bFGF, Instruchemie, the The Netherlands), and osteogenic medium was basic medium supplemented with $10 \mathrm{nM}$ dexamethasone (Sigma) and $10 \mathrm{mM}$-glycerophosphate (Sigma) (Luo et al., 2014).

Icaritin was added to $\mathrm{BM}$ and $\mathrm{OM}$ according to the saturated concentration of icaritin in culture medium with/without the presence of $\mathrm{CaP}$ ceramic discs (e.g., 2,000 and 4,300 ng/ml, as obtained from icaritin release test), thus both $\mathrm{BM}$ and $\mathrm{OM}$ containing $0,20,200,2,000$ and $4,300 \mathrm{ng} / \mathrm{ml}$ icaritin were prepared by first dissolving $5 \mathrm{mg}$ icaritin in $10 \mathrm{ml}$ methyl alcohol as the stock solution, followed by diluting this stock solution with either BM or OM. Specifically, additional methyl alcohol was added to each medium to keep the methyl alcohol concentration in all medium consistent.

\section{Human Bone Marrow Stromal Cells (hBMSC) Cultivation}

hBMSc (Passage 3, D307, Lonza) were expanded in cell culture flasks with GM under a humidified atmosphere of $5 \% \mathrm{CO}_{2}$ at $37^{\circ} \mathrm{C}$ until $80 \%$ confluence. Then GM was removed, and cells were harvested with $0.25 \%$ trypsin (Invitrogen, United Kingdom). 5,000 cells/well were seeded in 48 -well cell culture plates $(n=$ 5 per condition at each time point) and cultured with various medium prepared as described above. The medium was refreshed twice a week accordingly, and cells were harvested after 1, 4, 7, 14, and 21 days for further assays.

\section{Cell Morphology}

By day 14 , cells were rinsed once with $37^{\circ} \mathrm{C}$ phosphate-buffered saline (PBS) and fixed with $4 \%$ formaldehyde solution at room temperature for $10 \mathrm{~min}$. Afterwards, the fixation solution was removed and cells were rinsed again with PBS and stained with $1 \%$ methylene blue solution for $1 \mathrm{~min}$. Cell morphology was visualized using a Nikon SMZ800 stereomicroscope equipped with a Nikon camera.

\section{Calcium Deposition}

By day 14,21 , cells were rinsed once with $37^{\circ} \mathrm{C}$ phosphatebuffered saline (PBS) and fixed with $4 \%$ formaldehyde solution at room temperature for $10 \mathrm{~min}$. Afterwards, the fixation solution was removed, and cells were rinsed once with PBS, twice with distilled water and stained with $2 \%$ alizarin red staining for $1 \mathrm{~min}$. Excessive staining was rinsed twice with distilled water. Staining was visualized using a Nikon SMZ800 stereomicroscope equipped with a Nikon camera.

\section{Cell Proliferation and Osteogenic Differentiation Assay} By day $1,4,7,14,21$, cells were frozen at $-80^{\circ} \mathrm{C}$ for $24 \mathrm{~h}$ after rinsed once with $37^{\circ} \mathrm{C}$ phosphate-buffered saline (PBS). The proliferation of hBMSCs was analyzed with a CyQuant cell proliferation assay kit (Invitrogen). Briefly, to obtain the cell lysate, $150 \mu \mathrm{L}$ CyQuant lysis buffer was added to each well after cell culture plates were thawed at room temperature, followed by shooking at $400 \mathrm{rpm}$ for $5 \mathrm{~min}$. Then GR reagent was diluted 400 times by CyQuant lysis buffer. After $100 \mu \mathrm{L}$ diluted reagent was incubated with $100 \mu \mathrm{L}$ cell lysate for $5 \mathrm{~min}$ in the dark at room temperature, the fluorescence was detected using a spectrophotometer (Anthos Zenyth 3100) at wavelength of $480 \mathrm{~nm}$ for excitation and $520 \mathrm{~nm}$ for emission.

By using AttoPhos ${ }^{\circledR}$ AP fluorescent substrate system (catalog S1000, Promega), the amount of 2-[2-benzothiazoyl]-6hydroxybenzothiazole (BBT) produced during the reaction was used to present the Alkaline phosphatase (ALP) activity. Briefly, $90 \mu \mathrm{L}$ substrate reagent was incubated with $10 \mu \mathrm{L}$ cell lysate for $15 \mathrm{~min}$ in dark at room temperature. The fluorescence signal was then collected using a spectrophotometer (Anthos Zenyth 3100).

\section{Ectopic Implantation}

To study the influence of icaritin on bone formation, the icaritin-CaP discs (M0, M10, M50, M250, M1250) were evaluated in canine and rabbit ectopic models. All the animal experiments were followed the "Guidance Suggestions for the Care and Use of Laboratory Animals" by the National Science and Technology Committee of the People's Republic of China in order No. 2, 2006. Icaritin-CaP discs were implanted in the para-spinal muscles of five adult male beagles (body weight $14-17 \mathrm{~kg}$ ) for 8 weeks. The surgeries were performed under general anesthesia by abdominal injection of sodium pentobarbital (30 mg/kg body weight) under sterile conditions. Following the surgeries, penicillin $(40 \mathrm{mg} / \mathrm{kg}$ body weight) was injected intramuscularly for three consecutive days to prevent infection. After operation, all animals were allowed to bear full weight and received a normal diet. 

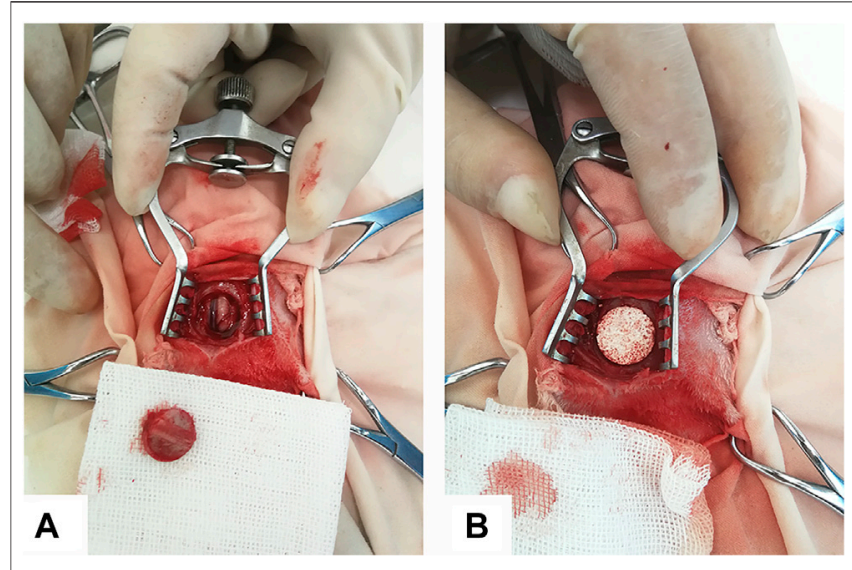

FIGURE 1 | Rabbit calvarial defect and implantation with porous scaffold. (A) $12.8 \mathrm{~mm}$ calvarial defect was created in rabbit calvarial midshaft. (B) CaP-based scaffold with size $12.8 \mathrm{~mm}$ was implanted into the defect region.

The animals were sacrificed via celiac injection of an excessive amount of pentobarbital sodium at week 8 . Implants were harvested with the surrounding tissues and fixed in $4 \%$ formaldehyde at $4^{\circ} \mathrm{C}$ for 1 week.

\section{Calvarial Defect Repair Surgery}

Thirty-six adult male New Zealand white rabbits (body weight $2.8-3.0 \mathrm{~kg}$ ) were used for this study. According to the results of Section 2.4, thirty-six rabbits were divided into two groups with M0 as the control group and M250 as the experimental group. All the animal experiments were followed the "Guidance Suggestions for the Care and Use of Laboratory Animals" by the National Science and Technology Committee of the People's Republic of China in order No. 2, 2006. The surgeries were performed under general anesthesia by abdominal injection of sodium pentobarbital (30 mg/kg body weight) under sterile conditions. After proper preparation, a midline sagittal incision in the scalp was made to expose the parietal bone and a critical-size $(12.8 \mathrm{~mm}$ in diameter) defect was created in the middle of parietal bone using a sterile drill (Figure 1A). After the bone was removed, the defects were grafted with porous icaritin-CaP ceramics (Figure 1B) and the incision was closed in layers using 4-0 Vicryl sutures. Following the surgeries, penicillin ( $40 \mathrm{mg} / \mathrm{kg}$ body weight) was injected intramuscularly for three consecutive days to prevent infection. After operation, all animals were allowed to bear full weight and received a normal diet.

At week 4 , week 8 , and week 12, each group of experimental animals was divided into an ink injection group and a non-ink injection group, with 3 animals in each group. To observe the growth of blood vessels, the animals in the ink injection group were injected with ink diluted with physiological saline (ratio 1:4) at a dose of $40 \mathrm{ml} / \mathrm{kg}$ after semi-anaesthesia with barbiturate sodium. The non-ink injection group was killed with an excess of pentobarbital sodium. All the defects with an additional $2 \mathrm{~mm}$ surrounding tissue were dissected from the host bone.
The samples of the ink injection group were fixed in $4 \%$ formaldehyde for histological quantification. After each sample in the non-ink injection group was divided into two halves, half was stored in liquid nitrogen for genetic testing, the other half was fixed in $4 \%$ formalin for histological observations.

\section{Quantitative Reverse Transcription-PCR (qRT-PCR)}

VEGF and FGF are crucial factors in angiogenesis. To determine the VEGF- and FGF-promoting effects of icaritin, qRT-PCR analyses were performed. All the samples were lysed in Trizol reagent (Ambion, USA), total RNA was extracted following the manufacturer's protocol (Chengdu Lilai Technology Co. Ltd., China) and reverse transcription was performed using RevertAid First Strand cDNA Synthesis Kit (Thermo Fisher Scientific, USA). Gene expressions were quantified using SYBR Green I PCR Kit (FastStart universal SYBR Green Master (Rox); Roche, Switzerland) in Real-Time PCR System (Thermo Fisher Scientific, USA). $\beta$-Actin was used as the housekeeping gene. Primers of the target genes including VEGF and FGF were listed in Table 1. Finally, the Real-time PCR reactin was run at 95 for $10 \mathrm{~min}$, followed by 95 for $30 \mathrm{~s}, 55$ for $30 \mathrm{~s}$ and 72 for $30 \mathrm{~s}$ for 45 cycles. The data were analysed using Thermo Scientific PikoReal software. The relative amounts of target genes normalized by $\beta$ Actin were calculated by the $2^{-\Delta \mathrm{CT}}$ method, where $\Delta \mathrm{C}_{\mathrm{T}}=\mathrm{C}_{\mathrm{T}}$, target ${ }^{-}$ $\mathrm{C}_{\mathrm{T}, \beta \text {-Actin. }}$

\section{Micro-CT}

Micro-CT (medium-resolution mode, isotropic voxel size: $15 \mu \mathrm{m}$; mCT 80, Scanco Medical AG, Bassersdorf, Switzerland) was used to evaluate new bone formation within the implants. The horizontal plane of all samples must be oriented perpendicular to the axis of the $\mathrm{X}$-ray beam. The $2 \mathrm{D}$ radiographic images were used to reconstruct and calculate corresponding bone volume fraction (bone volume/total volume, BV/TV) with Scanco software by thresholding $(n=3)$ (Chai et al., 2012; Ji et al., 2020).

\section{Histological Observations}

After fixation, all the harvested samples were dehydrated through a series of increasing ethanol concentrations and were subsequently embedded in methylmethacrylate. Three nondecalcified sections $(10-20 \mu \mathrm{m})$ from mid-defect regions of each sample were made by using a diamond saw microtome (Leica SP-1600, Germany) and stained with 1\% methylene blue (Sigma) and $0.3 \%$ basic fuchsin (Sigma) solutions for qualitative and quantitative histological observations.

\begin{tabular}{|c|c|c|}
\hline \multirow[t]{2}{*}{ Gene } & \multicolumn{2}{|c|}{ Primer sequences $\left(5^{\prime}-3^{\prime}\right)$} \\
\hline & Sense & Antisense \\
\hline$\beta$-Actin & GCTTCTAGGCGGACTGTTAG & CGAATAAAGCCATGCCAATCTC \\
\hline VEGF & TGGCAGAAGAAGGAGACAATAAA & GAAGATGTCCACCAAGGTCTC \\
\hline FGF & GCGACCCACACATCAAATTAC & GCAGTCTTCCATCTTCCTTCA \\
\hline
\end{tabular}



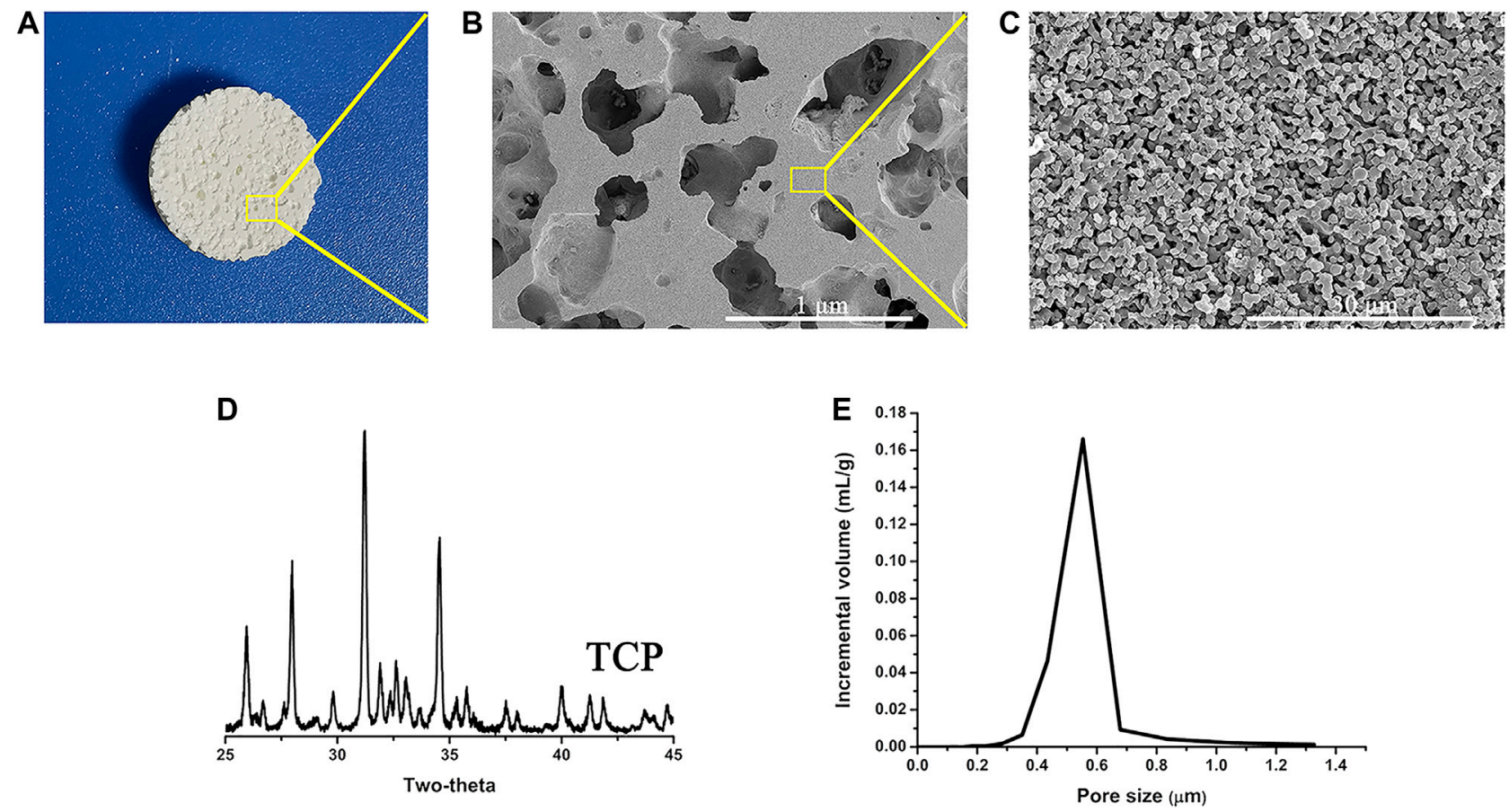

FIGURE 2 | Physical and chemical characteristics (A) CaP ceramic disc, (B) porous structure of CaP ceramic, (C) surface structure of CaP ceramic, (D) XRD pattern of the samples showing that the ceramic is B-TCP, and (E) micropore size distribution confirmed by Mercury intrusion.

To perform histomorphometry, the histological slides were scanned (Dimage Scan Elite 5400II, Konica Minolta photo Imaging Inc., Tokyo, Japan). After that, using Adobe Photoshop CS5 software, the region of interest (ROI), the ceramic materials $(\mathrm{M})$ and bone $(\mathrm{B})$ were quantified as the pixel values. Bone formation in the available space was quantitatively determined as follows: $\mathrm{B} \%=\mathrm{B}^{\star} 100 /(\mathrm{ROI}-\mathrm{M})$. Qualitative histology analysis of tissue formation (i.e. cell type, bone formation and presence of blood vessels) was performed by observing the slides at the light microscope (Nikon Eclipse E200, Tokyo, Japan).

\section{STATISTICS}

During the icaritin release experiment, multiple measures were performed on each sample, thus median of the measured data was used to reduce the effects of outliers. Mean was considered to represent bone formation as the sample number was limited. In the experiment of icaritin release, the data points were fitted with non-linear regression models to study the trend during time and were compared using one-way Wilcoxon signed rank-sum test. When means were considered, multiple comparisons were performed with one-way analysis of variance (ANOVA) followed by Bonferroni post-test comparisons. $p$-values lower than 0.05 were considered as statistically significant differences.

\section{RESULTS AND DISCUSSION}

\section{The CaP Ceramic Scaffold and Release Characteristics}

Figure 2B shows the porous structure of $\mathrm{CaP}$ ceramic, and the $\mathrm{XRD}$ pattern of $\mathrm{CaP}$ indicates that the ceramic is $\beta$-TCP (Figure 2D). Observing the microstructure size by SEM, CaP ceramic has a grain size of $0.95 \pm 0.20 \mu \mathrm{m}$ and a micropore size of $0.65 \pm 0.25 \mu \mathrm{m}$ (Figure 2C). Mercury intrusion indicates that the ceramic micropores range from $0.2-1.3 \mu \mathrm{m}$ with a peak of $0.55 \mu \mathrm{m}$ (Figure 2E). It can be seen from the Mercury intrusion data that the total porosity is $62 \%$ and the microporosity is $23 \%$.

Icaritin crystals could be observed on the surface of $\mathrm{CaP}$ ceramic and are rod-like with a diameter smaller than $10 \mu \mathrm{m}$ (Figures 3C,D). To detect icaritin with the HPLC-MS/MS system, optimization of precursor and product ions of the icaritin for MRM analysis by a triple quadrupole mass spectrometer with electrospray ionization was performed. The $\mathrm{m} / \mathrm{z}$ response observed was much better in the negative ionization mode than in the positive mode due to the phenolic hydroxyl group. The mass spectra showed deprotonated molecular ion [M-H]- peak at $385.0 \mathrm{~m} / \mathrm{z}$. On the basis of the production mass spectra, the major fragment ion was observed at 311.1 for icaritin (Figure 3A). A total ion chromatogram in full scan model was generated using standard icaritin solution, and icaritin could be separated and eluted at $5.50 \mathrm{~min}$ (Figure 3B). A good linearity was obtained for icaritin over the range from 2 to $500 \mathrm{ng} / \mathrm{ml}$ in culture medium (Figure 3E). 


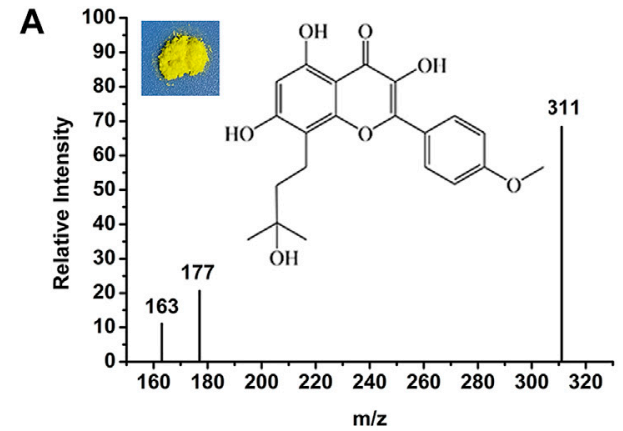

C

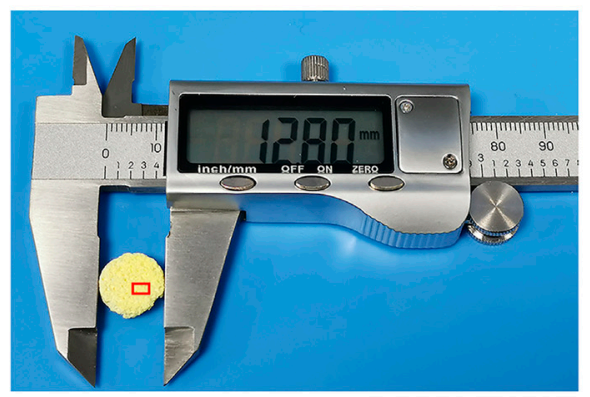

E

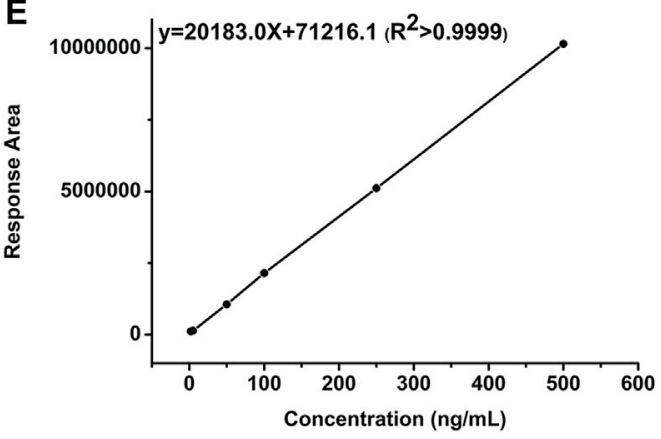

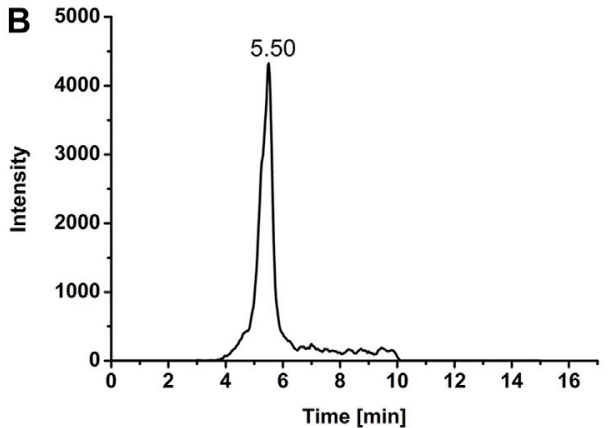

D

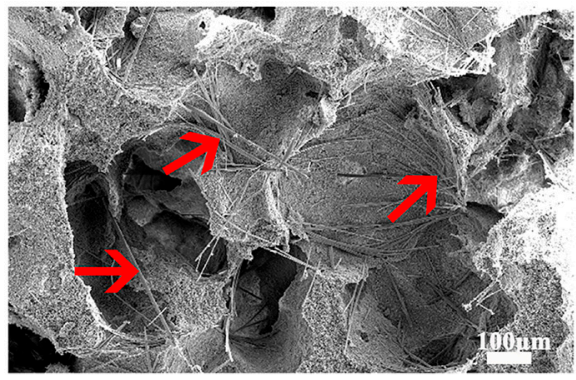

$\mathbf{F}$

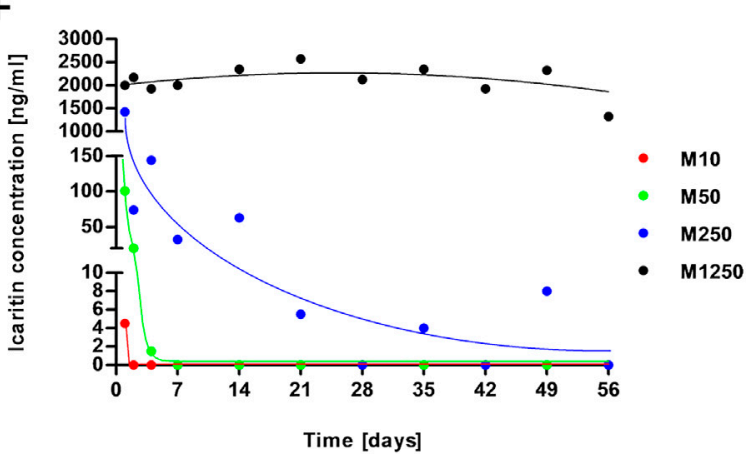

FIGURE 3 | Icaritin release and detection. (A) Chemical structure and full scan product ion of precursor ion of icaritin (the quantification ion at $\mathrm{m} / \mathrm{z} 311$ is generated via the loss of $\mathrm{C}_{4} \mathrm{H}_{9} \mathrm{O}$ from the precursor ion), (B) HPLC-MS/MS total ion chromatography of icaritin, (C) $12.8 \mathrm{~mm}$ CaP ceramic disc with icaritin, (D) needle-like icaritin on the surface of CaP ceramic (red arrow), (E) the icaritin calibration line between the concentrations from 2 to $500 \mathrm{ng} / \mathrm{ml}$ in culture medium, and (F) median values of the measured icaritin concentrations in the culture medium plotted against time (please note that the ordinate axis is split into three intervals for a better visualization of the results, which include all the data point measurements from all the materials).

According to the calibration line, the saturated icaritin concentrations at $37^{\circ} \mathrm{C}$ were $13.4 \pm 2.4 \mu \mathrm{g} / \mathrm{ml}$ in water and $4.3 \pm 0.1 \mu \mathrm{g} / \mathrm{ml}$ in culture medium respectively. The release of icaritin from the samples is summarized in Table 2 and illustrated in Figure 3F. No icaritin was detected in any of the controls (group M0). Initial burst releases, directly correlated with the icaritin content in the discs, were observed on the first day for all those samples containing icaritin (Figure 3F): the lesser icaritin incorporated in the $\mathrm{CaP}$ ceramic, the minor the intensity of the burst. Afterwards, all materials but M1250 had exponential release decays. The samples with the smaller icaritin amount M10 ceased to release already on the second day of experiment. A similar but delayed trend was observed for those samples containing $50 \mu \mathrm{g}$ of icaritin, which stopped releasing after one week. Discs with an initial load of $250 \mu \mathrm{g}$ icaritin were able to have
TABLE 2 | The quantities of total (cumulative) released and remaining icaritin in the samples after 56 days soaking.

\begin{tabular}{lcc} 
Sample & $\begin{array}{c}\text { Total icaritin released } \\
\text { after } \mathbf{5 6} \text { days }(\boldsymbol{\mu g})\end{array}$ & $\begin{array}{c}\text { Percentage of released } \\
\text { icaritin }\end{array}$ \\
\hline M0 & 0 & 0 \\
M10 & $0.052 \pm 0.065$ & $0.52 \%$ \\
M50 & $0.37 \pm 0.06$ & $0.74 \%$ \\
M250 & $5.54 \pm 0.58$ & $2.21 \%$ \\
M1250 & $69.80 \pm 11.91$ & $5.58 \%$ \\
\hline
\end{tabular}

a significant longer release decay rate as compared to M10 and M50. Samples M1250, with the highest icaritin initial content, kept a steady release during the whole experiment. The four materials, until they released icaritin, had significantly different 
release trends $(p<0.05$ for all data sets when compared in the periods of active release). The release measurements were corroborated by the observation that the majority of icaritin stayed in the discs after a 56-days soaking in culture medium (Table 2).

By its chemical nature, icaritin is theoretically not soluble in aqueous solution, and a low solubility of icaritin was seen in the study. In water, a saturated concentration of $13.4 \pm 2.4 \mu \mathrm{g} /$ $\mathrm{ml}$ was reached at $37^{\circ} \mathrm{C}$. The saturated concentration decreased to $4.3 \pm 0.1 \mu \mathrm{g} / \mathrm{ml}$ in the simulated body fluids (eg Dulbecco's Modified Eagle Medium plus foetal bovine serum), agreeing with the influence of proteins and ions on the solubility of chemicals (Galia et al., 1998). With a low solubility, a sustained release of icaritin was expected when it was loaded onto carriers. The sustained release was achieved in practice at the highest dose used in the study (e.g., M1250). The release profile of icaritin (Figure 3F and Table 2) indicated that icaritin was not only physically loaded into the $\mathrm{CaP}$ ceramic but also reacted chemically to $\mathrm{CaP}$. If only physical loading was there, the constant release of icaritin from M1250 should be around $4.3 \mu \mathrm{g} / \mathrm{ml}$ in simulated body fluids, while it was $2.0 \mu \mathrm{g} / \mathrm{ml}$ in practice. Moreover, if only physical loading was there, a constant release could have happened in the case of $250 \mu \mathrm{g}$ icaritin/disc as well, since the total theoretical release of icaritin in the tested time frame was $141.9 \mu \mathrm{g}$ (11-time points with the theoretical constant release of $4.3 \mu \mathrm{g} / \mathrm{ml}$ in $3 \mathrm{ml}$ ), less than $250 \mu \mathrm{g}$ icaritin loaded. As shown in Table 2, the majority of icaritin was retained in $\mathrm{CaP}$ ceramics after a 56-days soaking in simulated body fluids, further supporting the chemical reaction of icaritin to the $\mathrm{CaP}$ ceramic. The exact reaction of icaritin to $\mathrm{CaP}$ ceramic needs to be further addressed with studies, while it is likely that icaritin could react to calcium phosphate through coordination bond with $\mathrm{Ca}^{2+}$ ions, meaning that there might exist some dynamic equilibria involving icaritin and $\mathrm{Ca}^{2+}$ ions (Huskens and Sherry, 1998). As such, the saturated concentration of icaritin further decreased to $2.0 \mu \mathrm{g} / \mathrm{ml}$ in simulated body fluids when loaded into the CaP ceramic (Figure 3F, M1250).

\section{Influence of Icaritin on hBMSCs}

Bearing the possible reaction between icaritin and $\mathrm{CaP}$ ceramic after loading, icaritin released from $\mathrm{CaP}$ ceramic discs into simulated body fluids dose-dependently (Figure 3F). Using the saturated concentration in culture medium and dilutions of the highest icaritin release from $\mathrm{CaP}$ ceramic into culture medium (e. g., $2 \mu \mathrm{g} / \mathrm{ml}$ ), the influence of icaritin on the activities of human bone marrow stromal cells was evaluated. A morphological change from spindle to polygon shape was observed by day 14 with the increasing concentration of icaritin (Figure 4B), regardless of the culture media used (BM vs OM). Specifically, with the increasing of icaritin up to $2,000 \mathrm{ng} / \mathrm{ml}$, hBMSCs became more spread and larger, indicating their tailored cell fate in the presence of icaritin. In both BM and OM 2,000 ng/ml icaritin inhibited the proliferation of hBMSCs while $4,300 \mathrm{ng} / \mathrm{ml}$ icaritin showed severe cytotoxicity that it induced apoptosis, where not much cells but crystals of un-dissolved icaritin were observed under the light microscope instead of living cells (Figure $4 \mathbf{B}$, $4,300 \mathrm{ng} / \mathrm{ml}$ ).

Calcium deposition was only observed in OM group but not in BM (Figure 4A). By day 14 and 21 the calcium deposition was seen in $20 \mathrm{ng} / \mathrm{ml}$ and $200 \mathrm{ng} / \mathrm{ml}$, and occasionally seen in $2,000 \mathrm{ng} / \mathrm{ml}$ and $4,300 \mathrm{ng} / \mathrm{ml}$.

hBMSCs proliferated and peaked by day 14 in both BM and OM, except in those supplemented with $2000 \mathrm{ng} / \mathrm{ml}$ icaritin or more (Figure 4D). With the presence of osteogenic factor (e.g., DEX), cells, in the means of DNA content, were not much different in number among the groups of 0,20 , and $200 \mathrm{ng} /$ $\mathrm{ml}$, while without osteogenic factor, cells were much more in number in groups of 20 and $200 \mathrm{ng} / \mathrm{ml}$ as compared to $0 \mathrm{ng} / \mathrm{ml}$ control (Figure 4D).

Given the fact that a high dose of icaritin was harmful to the cells, ALP assay was not applied to the samples of 4,300 ng icaritin $/ \mathrm{ml}$. The ALP expression of hBMSCs is relatively low in $\mathrm{BM}$ as compared to OM group regardless of the concentration of icaritin (Figure 4C). Moreover, ALP/DNA in cells cultured with $\mathrm{BM}$ did not increase with time from Day 7 to Day 21, while ALP/DNA increased from Day 7 to Day 21 (Figure 4C) in cells cultured with OM. Furthermore, at day 21, ALP/DNA was up to the concentration of icaritin, with higher ALP/DNA at higher concentration of icaritin (at the dose range of $0-200 \mathrm{ng} / \mathrm{ml}$ ) (Figure 4C).

So, at the saturated concentration in culture medium $(4.3 \mu \mathrm{g} / \mathrm{ml})$, icaritin was harmful to hBMSCs and caused cell death (Figures 4B,D). At the highest release from $\mathrm{CaP}$ ceramic $(2 \mu \mathrm{g} / \mathrm{ml}$ from M1250), icaritin inhibited cell proliferation (Figures 3B, 4D). At the concentration between $0.02-0.20 \mu \mathrm{g} / \mathrm{ml}$, icaritin enhanced proliferation of hBMSCs in the absence of osteogenic factor (eg Dex), while it did not affect hBMSC proliferation when dex was used in the culture media (Figures 4B,D). At the concentration of $0.02-0.20 \mu \mathrm{g} / \mathrm{ml}$, icaritin did not enhance osteogenic differentiation of hBMSCs when Dex was not used in the culture medium, as indicated in both mineralization assay (Figure 4A) and ALP/DNA assay (Figure 4C), with the presence of Dex in the culture media, icaritin did enhance osteogenic differentiation of hBMSCs (Figures 4A,C). The data agreed with the previous findings that at certain dosages icaritin did not affect bone marrow stromal cell proliferation, but enhanced ALP production and mineralization while at high doses, meaning that icaritin enhances osteogenic function of readily differentiated osteogenic cells but does not induce osteogenic differentiation of osteoprogenitors or stems cells in vitro.

\section{Influence of Icaritin in Ectopic Bone Formation}

When icaritin-loaded $\mathrm{CaP}$ ceramic discs were implanted intramuscularly in canine for 8 weeks, the dose-dependent influence of icaritin on bone formation was seen (Figures 5, 6). The samples $(n=5)$ harvested from all animals were available for histological evaluation and histomorphometry. The least bone formation was observed in group M1250 and most bone formation occurred in groups M50 and M250 (Figures 5, 6). 

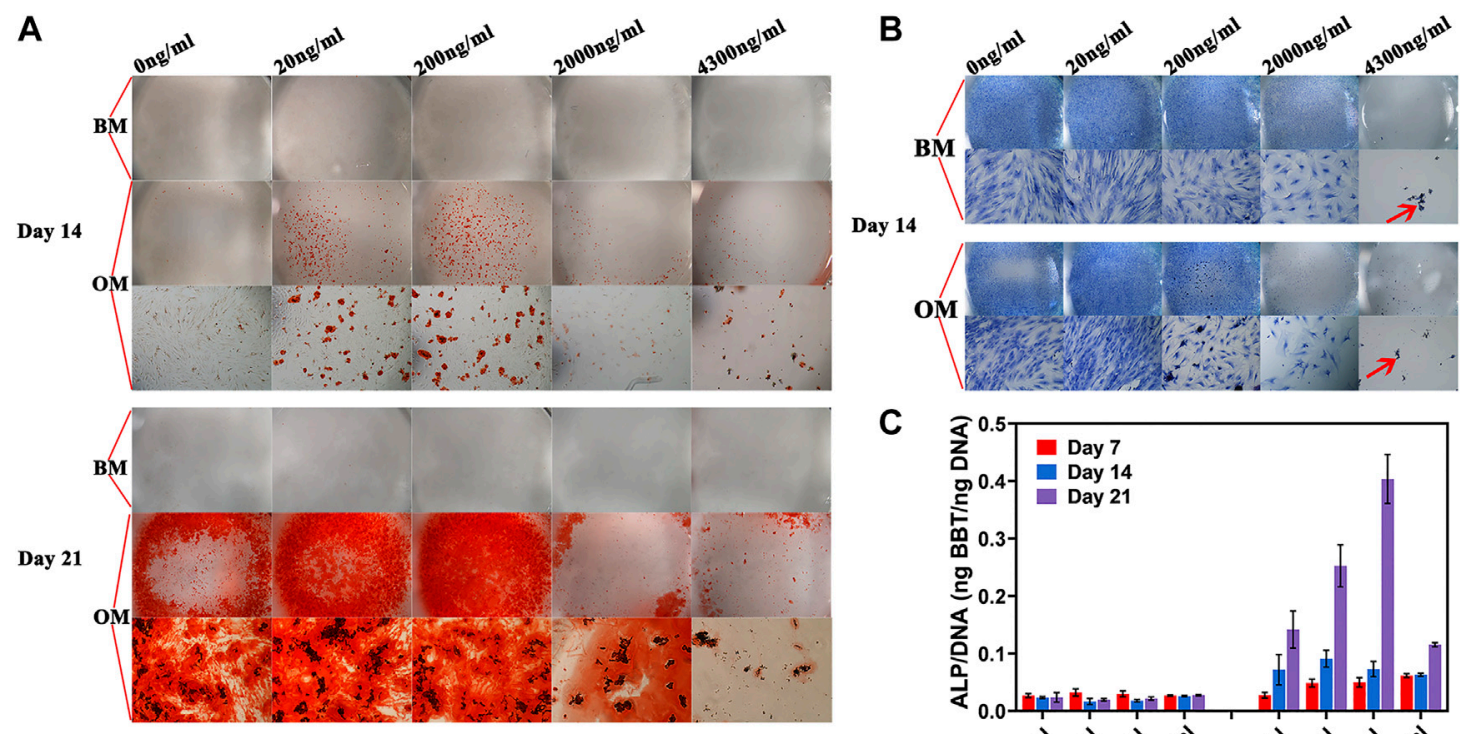

\section{C}

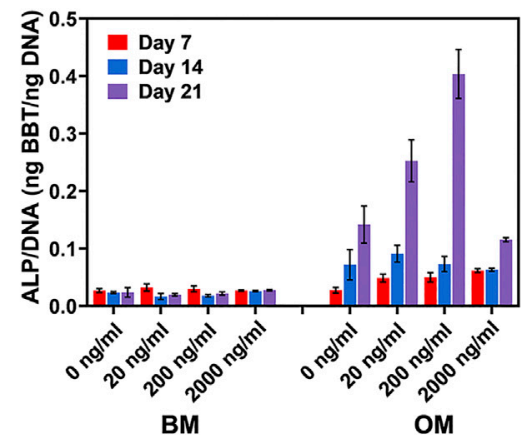

D

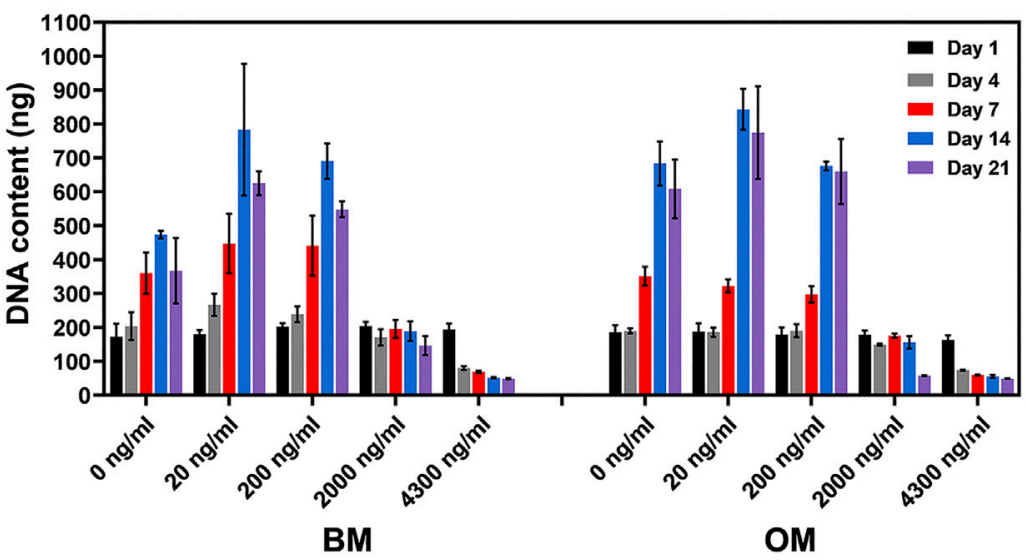

FIGURE 4 | hBMSC proliferation and osteogenic differentiation in different culture medium. (A) Mineralization and (B) cell morphology of hBMSC in basic medium and osteogenic medium as the function of icaritin dose (red arrow stands for un-dissolved icaritin crystals), (D) hBMSC proliferation, and (C) osteogenic differentiation in $\mathrm{BM}$ and $\mathrm{OM}$ as a function of icaritin dose.

The addition of $10 \mu \mathrm{g}$ did not improve bone formation as compared to the control group. Loading 50 or $250 \mu \mathrm{g}$ icaritin significantly increased the bone formation in the $\mathrm{CaP}$ ceramic. When icaritin was added in a high dose (i.e., $1,250 \mu \mathrm{g}$ ), it had an inhibitory effect on the ectopic bone formation of the ceramic.

Both the icaritin liberated from $\mathrm{CaP}$ ceramic and the icaritin that stayed in the $\mathrm{CaP}$ ceramic may have played biological roles in vivo. At the concentrations of $10^{-7}-10^{-5} \mathrm{~mol} / \mathrm{L}$ (i.e., $0.0385-3.85 \mu \mathrm{g} / \mathrm{ml}$ ), icaritin enhanced the differentiation and proliferation of osteoblasts in vitro (Huang et al., 2007). If only the icaritin liberated from $\mathrm{CaP}$ ceramic affected bone formation, then bone formation may not have been enhanced in the $50 \mu \mathrm{g}$ icaritin group since the release of icaritin stopped 7 days after immersing in simulated body fluid, while it was known that inductive bone formation in canine muscle normally starts
4 weeks after implantation (Yuan et al., 2006). Similarly, if only the icaritin released from $\mathrm{CaP}$ ceramic had an essential contribution, then bone formation should have been enhanced in the $1,250 \mu \mathrm{g}$ group in dogs because the $2 \mu \mathrm{g} / \mathrm{ml}$ concentration was still within the reported interval of efficient icaritin concentration to enhance bone formation. Instead, it was found in this study that the bone formation was inhibited in the $1,250 \mu \mathrm{g}$ group. The inhibited bone formation in the $1,250 \mu$ g group could be attributed to the cytotoxicity of icaritin at high dosages (Chen et al., 2012; Chen et al., 2013).

Based on the in vitro results, it is clear that icaritin does not have osteoinductive potential as the bone morphogenetic proteins have (Chen et al., 2012), icaritin could thus only affect bone formation in an environment where bone formation was readily present. In other words, icaritin enhanced a bone formation process that was already triggered by the submicron scaled 

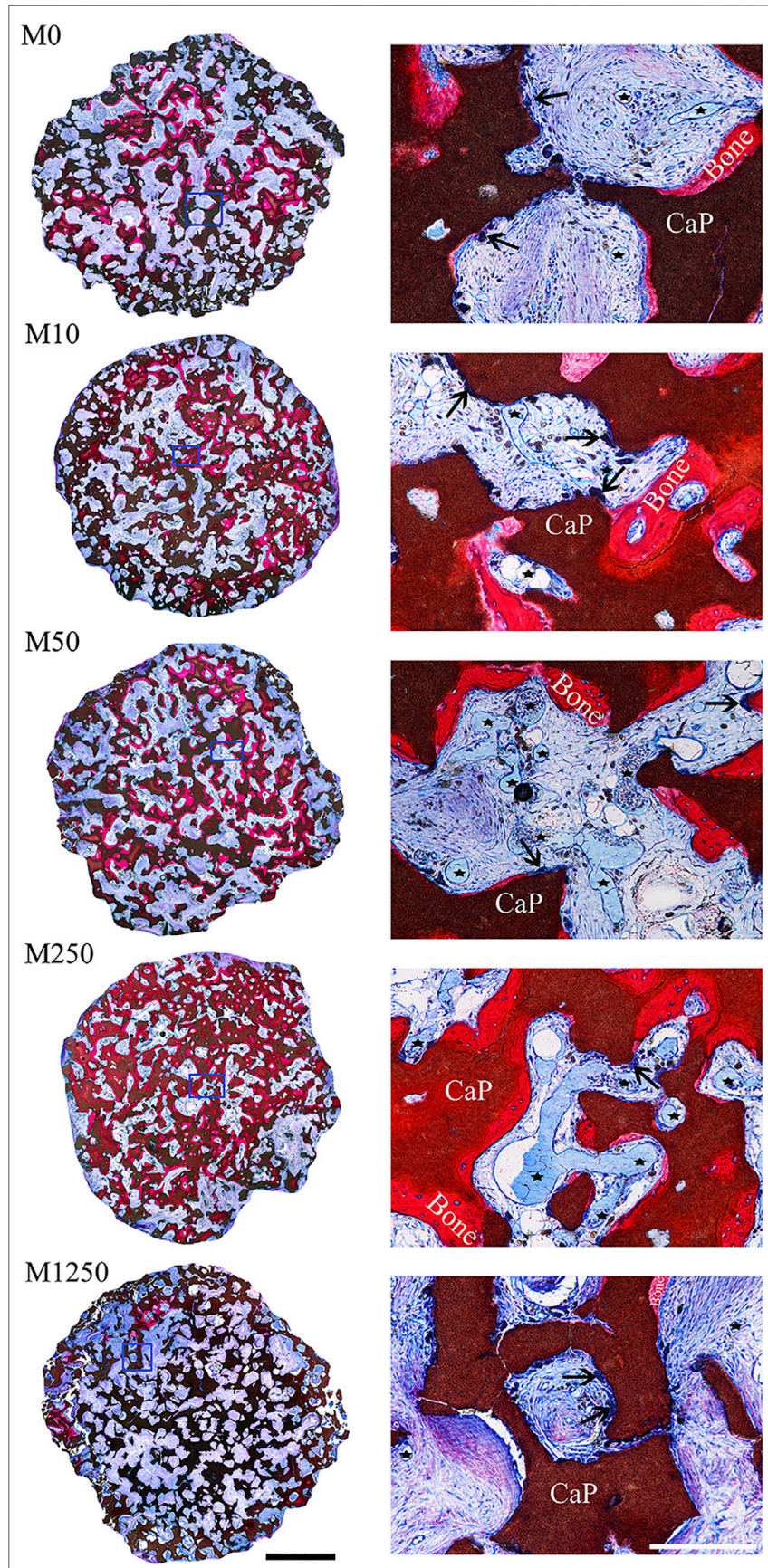

FIGURE 5 | Tissue response to icaritin-containing CaP discs implanted in muscle of dogs for 8 weeks. Left, histological overviews of M0, M10, M50, M250 and M1250 (scale = 2 mm); Right, detailed light microscopic images of the selected region in the histological overviews (scale $=250 \mu \mathrm{m})$ (undecalcified sections; methylene blue and basic fuchsin staining; Bone: bone tissue; CaP: CaP ceramic; star: blood vessel; arrow head: multinucleate giant cells).

surfaces of the CaP ceramic implanted in this study in dogs. Via protein adsorption (Yuan et al., 2010; Zhang et al., 2014), surface mineralization (Zhang et al., 2015; Murr, 2019), ion-release (Yuan et al., 2010; Zhang et al., 2014; Zhang et al., 2015) and surface topographic cues (Zhang et al., 2015), submicron surface structured calcium phosphate ceramic generates an osteogenic environment to which mesenchymal stem cells respond and undergo osteogenic differentiation to form bone. Together with the osteogenic environment generated by the submicron surface structured calcium phosphate ceramic, icaritin enhanced the function of mesenchymal stem cells and bone formation was eventually enhanced.

\section{Calvarial Defect Repair Evaluation}

Studies have shown that angiogenesis plays a vital role in bone tissue repair process, and enhanced neovascularization accelerates bone formation (Karageorgiou and Kaplan, 2005; Oates et al., 2007; Barralet et al., 2009). As observed in Figure 5, an increase in blood vessels amount was seen from M0 to M250, the pores of all implants were occupied by soft tissues including blood vessels, indicating enhanced bone formation was associated with higher density of blood vessels. It has been shown that stem cells could reach to the osteoinductive calcium phosphate ceramic via blood circulation (Song et al., 2013). It is likely that icaritin first enhanced angiogenesis from which stem cells responsible for inductive bone formation could be recruited. When the stem cells were differentiated by $\mathrm{CaP}$ ceramic, icaritin enhanced the function of such differentiated cells to form bone.

It is thus worthy of performing studies to test the functionality of icaritin-containing osteoinductive $\mathrm{CaP}$ ceramic in bone regeneration in orthopedic sites. The in vivo bone regeneration effect of samples was evaluated by micro-CT and histological analysis. The 3D images of Micro-CT reconstruction in Figure 7A demonstrate newly formed bone within implants at week 4,8 , and 12 , and the quantitative data of new bone volume $(\mathrm{BV} / \mathrm{TV})$ within the implants was measured by the $2 \mathrm{D}$ radiographs. Compared with M0 group, there was a significant

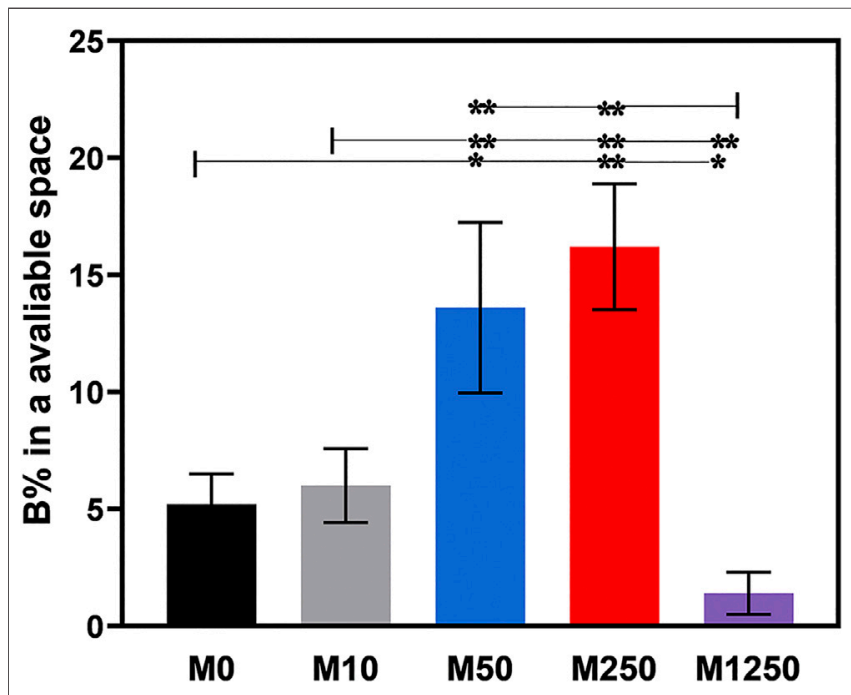

FIGURE 6 | Bone formation in the available space (\%) of samples harvested from dogs (statistically significant differences are marked by $\left.{ }^{\star} p<0.05,{ }^{* *} p<0.01\right)$ 


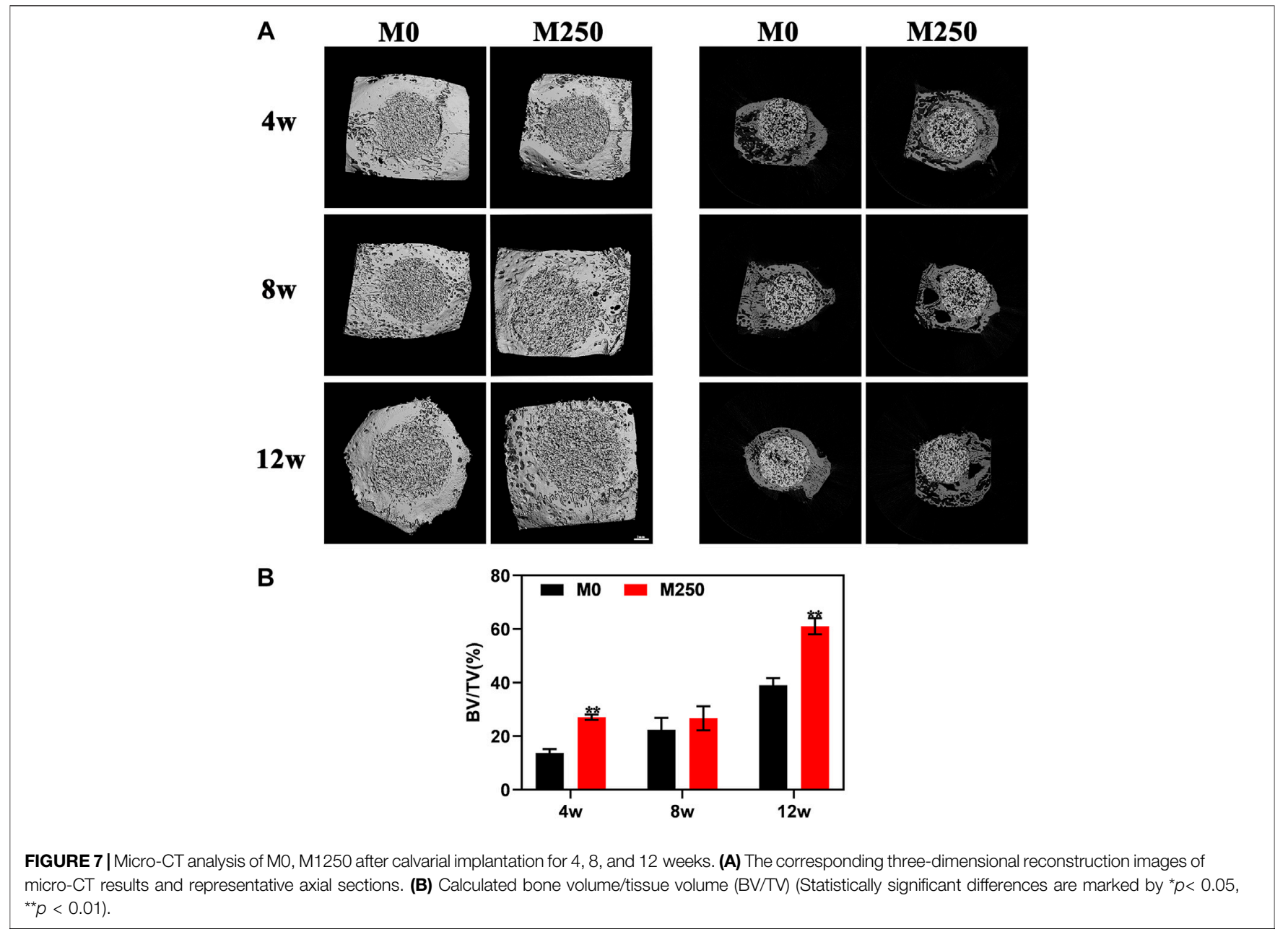

increase in BV/TV in M250 group at week 4 (Figure 7B) $(p<0.01, \mathrm{n}=3)$. After 8 weeks post implantation, although the growth rate of the M250 group is lower than that of M0 group, the BV/TV of the M250 group is still higher than that of M0 group (Figure 7B). At week 12, the BV/TV in M250 group was significantly higher than that of M0 group (Figure 7B) $(p<$ $0.01, \mathrm{n}=3$ ). And the BV/TV of all groups at week 12 was higher than that of week 8 .

Figures 8 and 10 display the histological manifestations in methylene blue and basic fuchsin stains after implantation for 4,8 and 12 weeks, respectively. Observed from the axial and sagittal direction, the newly formed bone tissue became evident at week 4 with limited bony bridging along the radial border of the bone defect (Figure 8A, Figure 8 $\mathbf{a}_{2}$, Figure 8B, Figure $8 b_{2}$, Figure $10 A$, Figure $10 a_{1}$, Figure $10 a_{2}$, Figure $10 \mathrm{~B}$, Figure $10 \mathrm{~b}_{1}$, Figure $10 \mathrm{~b}_{2}$ ). Compared with week 4 , at week 8 , more new bone tissue in both groups grew into the scaffold, along the radial direction (Figure 8C, Figure 8D, Figure 10C, Figure 10D). Obviously, at week 12, the new bone tissue of the M250 group have almost penetrated the entire material (Figure 8F, Figure 10F). Serial graphs reveal how new bone first originates at the resident bone edges from where it proceeds first along the external margins of the scaffold implant and then towards the center of the scaffold. Quantitative analysis (Figure 9) shows that at week 4, week 8 and week 12, the M250 group had more new bone in the defect compared to the M0 group. At week 4 and week 8 , both groups had isolated new bone islands along the radial margins and within the pores of the scaffolds (Figure $\mathbf{8} a_{1}$, Figure $\mathbf{8} b_{1}$, Figure $\mathbf{8 c}_{1}$, Figure $\mathbf{8} \mathbf{d}_{1}$ ). The newly formed bone within the scaffold was seen to have remodelled towards mature lamellar bone with osteocytes within lacunae embedded in the bone matrix.

Newly formed vessels within the scaffold pores became visible in first 4 weeks in both groups (Figures 11A,B), and decreased from the next 4 weeks (Figures 11C,D,G-I). Quantitatively, compared with the M0 scaffold groups, the M250 scaffold group showed many more new vessels in terms of vessel volume, VEGF and FGF expression within bone defects at week 4 , week 8 and week $12(n=3$, Figures $11 \mathrm{G}-\mathrm{I})$.

Animal experiments results showed that icaritin significantly improve the ingrowth of blood vessel with a large amount of bone tissue penetrated the entire scaffold at 


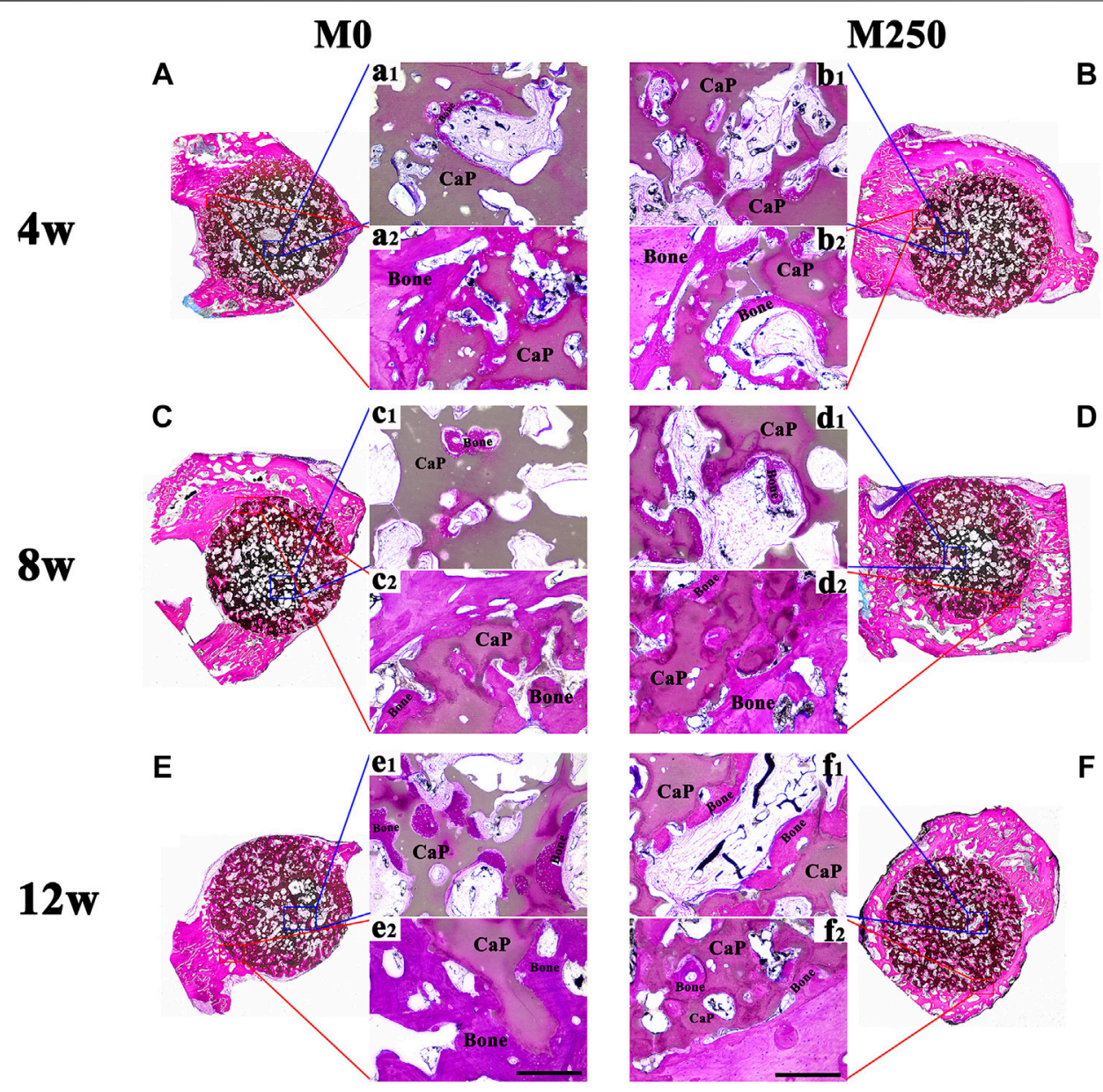

FIGURE 8| The axial direction histological observation of M0 and M250 after calvarial implantation for 4, 8 and 12 weeks (A), (C), (E) The histological overviews of M0 (B), (D), (F) The histological overviews of M250 $\left(a_{1}\right)\left(c_{1}\right)\left(e_{1}\right)$ New bone islands within the M0 scaffolds pores $\left(b_{1}\right)\left(d_{1}\right)\left(f_{1}\right)$ New bone islands within the M250 scaffolds pores $\left(a_{2}\right)\left(c_{2}\right)\left(e_{2}\right)$ New bone tissue crawl into the MO scaffolds $\left(b_{2}\right)\left(d_{2}\right)\left(f_{2}\right)$ New bone tissue crawl into the M250 scaffolds $(\mathrm{scale}=500 \mu \mathrm{m})$, Bone: bone tissue; CaP: CaP ceramic.

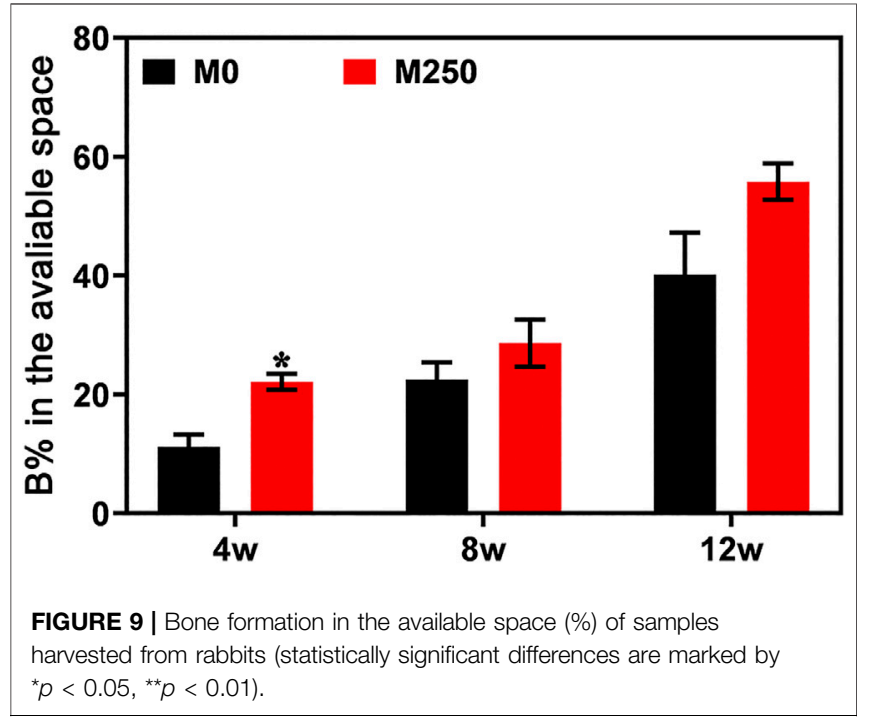

week 12, when compared with M0 group (Figures 7-10). And compared with week 4 , although the number of blood vessels in M250 decreased at week 8, the number of connected blood vessels increased, accompanied by more bone regeneration (Figure 11). At week 12, the blood vessel volume of the M250 group was still higher than that of the M0 group (Figures 11E-I). These results support other evidence that icaritin enhanced angiogenesis and bone formation in rabbits when icaritin was incorporated into PLGA/TCP composites (Chen et al., 2012; Chen et al., 2013), indicating a possible link between angiogenesis and bone formation which was described in the phenomenon of osteoinduction caused by icaritin-loaded $\mathrm{CaP}$ ceramic.

\section{CONCLUSION}

When loaded into submicron surface structured $\mathrm{CaP}$ ceramics, icaritin was chemically retained and released 

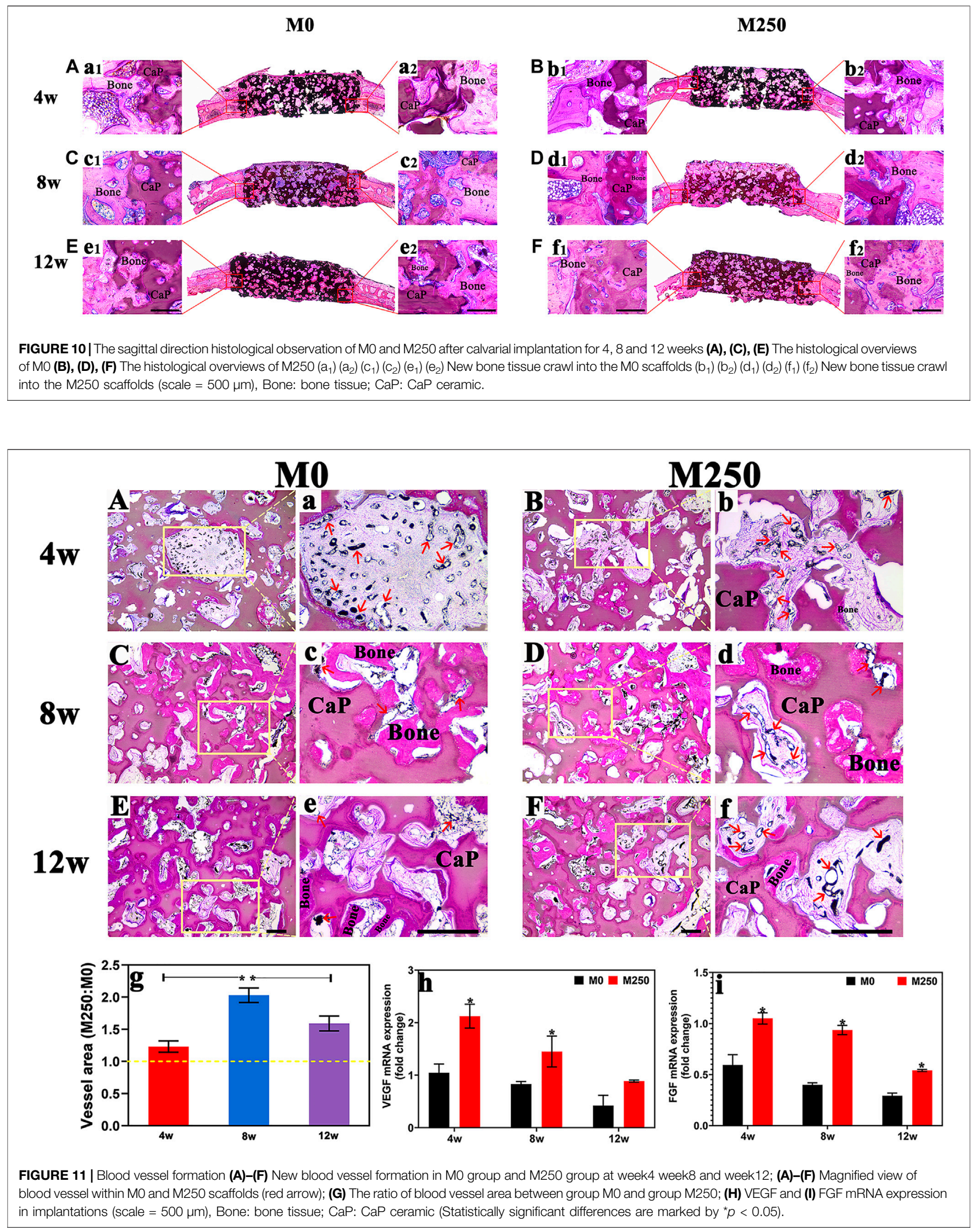
dose-dependently. With its release, icaritin enhanced osteogenesis of bone marrow stromal cells in vitro with the presence of osteogenic factors and enhanced bone formation initiated by submicron surface structured $\mathrm{CaP}$ ceramic in intramuscular implantation. Due to enhanced bone formation by icaritin, icaritin-containing osteoinductive $\mathrm{CaP}$ ceramic scaffolds presented the desired osteogenic and angiogenic potential, as evidenced in a rabbit calvarial defect model. The combination of icaritin and submicron surface structured $\mathrm{CaP}$ ceramics could thus be a novel strategy for bone regeneration.

\section{DATA AVAILABILITY STATEMENT}

The raw data supporting the conclusions of this article will be made available by the authors, without undue reservation.

\section{REFERENCES}

Barralet, J., Gbureck, U., Habibovic, P., Vorndran, E., Gerard, C., and Doillon, C. J. (2009). Angiogenesis in calcium phosphate scaffolds by inorganic copper ion release. Tissue Eng. 15 (7), 1601-1609. doi:10.1089/ten.tea.2007.0370

Bohner, M., Galea, L., and Doebelin, N. (2012). Calcium phosphate bone graft substitutes: failures and hopes. J. Eur. Ceram. Soc. 32 (11), 2663-2671. doi:10. 1016/j.jeurceramsoc.2012.02.028

Chai, Y. C., Kerckhofs, G., Roberts, S. J., Van Bael, S., Schepers, E., Vleugels, J., et al. (2012). Ectopic bone formation by $3 \mathrm{D}$ porous calcium phosphate-Ti6Al4V hybrids produced by perfusion electrodeposition. Biomaterials 33 (16), 4044-4058. doi:10.1016/j.biomaterials.2012.02.026

Chen, S. H., Wang, X. L., Xie, X. H., Zheng, L. Z., Yao, D., Wang, D. P., et al. (2012). Comparative study of osteogenic potential of a composite scaffold incorporating either endogenous bone morphogenetic protein-2 or exogenous phytomolecule icaritin: an in vitro efficacy study. Acta Biomater. 8 (8), 3128-3137. doi:10.1016/j.actbio.2012.04.030

Chen, S. H., Lei, M., Xie, X. H., Zheng, L. Z., Yao, D., and Wang, X. L. (2013). PLGA/TCP composite scaffold incorporating bioactive phytomolecule icaritin for enhancement of bone defect repair in rabbits. Acta Biomater. 9 (5), 6711-6722. doi:10.1016/j.actbio.2013.01.024

Damien, C. J., and Parsons, J. R. (1991). Bone graft and bone graft substitutes: a review of current technology and applications. J. Appl. Biomater. 2 (3), 187-208. doi:10.1002/jab.770020307

Davison, N. L., Gamblin, A. L., Layrolle, P., Yuan, H., de Bruijn, J. D., and Barrère-de Groot, F. (2014a). Liposomal clodronate inhibition of osteoclastogenesis and osteoinduction by submicrostructured beta-tricalcium phosphate. Biomaterials 35(19), 5088-5097. doi:10.1016/j.biomaterials.2014.03.013

Davison, N. L., Luo, X., Schoenmaker, T., Everts, V., Yuan, H., Barrère-de Groot, F., et al. (2014b). Submicron-scale surface architecture of tricalcium phosphate directs osteogenesis in vitro and in vivo. Eur. Cell. Mater. 27, 281-287. doi:10. 22203/ecm.v027a 20

Galia, E., Nicolaides, E., Hörter, D., Löbenberg, R., Reppas, C., and Dressman, J. B. (1998). Evaluation of various dissolution media for predicting in vivo performance of class I and II drugs. Pharm. Res. (N. Y.) 15 (5), 698-705. doi:10.1023/a:1011910801212

Habibovic, P., van der Valk, C. M., van Blitterswijk, C. A., De Groot, K., and Meijer, G. (2004). Influence of octacalcium phosphate coating on osteoinductive properties of biomaterials. J. Mater. Sci. Mater. Med. 15 (4), 373-380. doi:10.1023/b:jmsm.0000021104.42685.9f

Hench, L. L., and Wilson, J. (1984). Surface-active biomaterials. Science 226 (4675), 630-636. doi:10.1126/science.6093253

Huang, J., Yuan, L., Wang, X., Zhang, T. L., and Wang, K. (2007). Icaritin and its glycosides enhance osteoblastic, but suppress osteoclastic, differentiation and activity in vitro. Life Sci. 81 (10), 832-840. doi:10.1016/j.lfs.2007.07.015

\section{ETHICS STATEMENT}

The animal study was reviewed and approved by The First Affiliated Hospital of Chongqing Medical University.

\section{AUTHOR CONTRIBUTIONS}

$\mathrm{HP}$ and JL performed the experiments, analyzed and interpreted the results. YX performed the animal experiments. GL acquired the research operating funding, advised on the conception and conduction of the experiments and data analysis.

\section{ACKNOWLEDGMENTS}

The authors thank Science and Technology Project of Sichuan Province (2018FZ0108) for providing funding for this research.

Huskens, J., and Sherry, A. D. (1998). Co-ordination chemistry and molecular mechanics study of the magnesium(II) and calcium(II) complexes of trisubstituted 1,4,7-triazacyclononane derivatives. J. Chem. Soc. Dalton Trans. 5 (1), 177-184. doi:10.1039/a703640j

Ji, M., Chen, H., Yan, Y., Ding, Z., Ren, H., and Zhong, Y. (2020). Effects of tricalcium silicate/sodium alginate/calcium sulfate hemihydrate composite cements on osteogenic performances in vitro and in vivo. J. Biomater. Appl. 34 (10), 1422-1436. doi:10.1177/0885328220907784

Karageorgiou, V., and Kaplan, D. (2005). Porosity of 3D biomaterial scaffolds and osteogenesis. Biomaterials 26 (27), 5474-5491. doi:10.1016/j.biomaterials.2005. 02.002

Lai, X., Ye, Y., Sun, C., Huang, X., Tang, X., Zeng, X., et al. (2013). Icaritin exhibits anti-inflammatory effects in the mouse peritoneal macrophages and peritonitis model. Int. Immunopharm. 16 (1), 41-49. doi:10.1016/j. intimp.2013.03.025

Lee, K. G., Lee, K. S., Kang, Y. J., Hwang, J. H., Lee, S. H., Park, S. H., et al. (2018). Rabbit calvarial defect model for customized 3D-printed bone grafts. Tissue Eng. C Methods. 24 (5), 255-262. doi:10.1089/ten.TEC.2017.0474

Luo, X., Barbieri, D., Davison, N., Yan, Y., de Bruijn, J. D., and Yuan, H. (2014). Zinc in calcium phosphate mediates bone induction: in vitro and in vivo model. Acta Biomater. 10(1), 477-485. doi:10.1016/j.actbio.2013.10.011

McMillan, A., Nguyen, M. K., Gonzalez-Fernandez, T., Ge, P., Yu, X., Murphy, W. L., et al.(2018). Dual non-viral gene delivery from microparticles within 3D high-density stem cell constructs for enhanced bone tissue engineering. Biomaterials 161, 240-255. doi:10.1016/j.biomaterials.2018.01.006

Murr, L. E. (2019). Strategies for creating living, additively manufactured, opencellular metal and alloy implants by promoting osseointegration, osteoinduction and vascularization: an overview. J. Mater. Sci. Technol. 35 (2), 231-241. doi:10.1016/j.jmst.2018.09.003

Oates, M., Chen, R., Duncan, M., and Hunt, J. A. (2007). The angiogenic potential of three-dimensional open porous synthetic matrix materials. Biomaterials 28 (25), 3679-3686. doi:10.1016/j.biomaterials.2007.04.042

Peng, S., Zhang, G., Zhang, B. T., Guo, B., He, Y., Bakker, A. J., et al. (2013). The beneficial effect of icaritin on osteoporotic bone is dependent on the treatment initiation timing in adult ovariectomized rats. Bone 55 (1), 230-240. doi:10. 1016/j.bone.2013.02.012

Qin, L., Zhang, G., Sheng, H., Wang, X. L., Wang, Y. X., Yeung, K. W., et al. (2008). Phytoestrogenic compounds for prevention of steroid-associated osteonecrosis. J. Musculoskelet. Neuronal Interact. 8 (1), 18-21. doi:10.1007/978-3-540-45456$4 \_38$

Song, G., Habibovic, P., Bao, C., Hu, J., van Blitterswijk, C. A., Yuan, H., et al. (2013). The homing of bone marrow MSCs to non-osseous sites for ectopic bone formation induced by osteoinductive calcium phosphate. Biomaterials 34 (9), 2167-2176. doi:10.1016/j.biomaterials.2012.12.010

van Dijk, L. A., Duan, R., Luo, X., Barbieri, D., Pelletier, M., Christou, C., et al. (2018). Biphasic calcium phosphate with submicron surface topography in an 
Ovine model of instrumented posterolateral spinal fusion. JOR Spine. 1 (4), e1039. doi:10.1002/jsp2.1039

Xu, S., Lin, K., Wang, Z., Chang, J., Wang, L., Lu, J., et al. (2008). Reconstruction of calvarial defect of rabbits using porous calcium silicate bioactive ceramics. Biomaterials 29 (17), 2588-2596. doi:10.1016/j.biomaterials.2008.03.013

Yao, D., Xie, X. H., Wang, X. L., Wan, C., Lee, Y. W., and Chen, S. H. (2012). Icaritin, an exogenous phytomolecule, enhances osteogenesis but not angiogenesis-an in vitro efficacy study. PLoS One. 7 (8), e41264. doi:10. 1371/journal.pone.0041264

Yuan, H., van Blitterswijk, C. A., de Groot, K., and de Bruijn, J. D. (2006). A comparison of bone formation in biphasic calcium phosphate (BCP) and hydroxyapatite (HA) implanted in muscle and bone of dogs at different time periods. J. Biomed. Mater. Res. 78 (1), 139-147. doi:10.1002/jbm.a. 30707

Yuan, H., Fernandes, H., Habibovic, P., de Boer, J., Barradas, A. M., and de Ruiter, A. (2010). Osteoinductive ceramics as a synthetic alternative to autologous bone grafting. Proc. Natl. Acad. Sci. U.S.A. 107 (31), 13614-13619. doi:10.1073/pnas. 1003600107

Zhang, G., Qin, L., and Shi, Y. (2007). Epimedium-derived phytoestrogen flavonoids exert beneficial effect on preventing bone loss in late postmenopausal women: a 24-month randomized, double-blind and placebo-controlled trial. J. Bone Miner. Res. 22 (7), 1072-1079. doi:10.1359/ jbmr.070405

Zhang, G., Pan, X. H., Xie, X. H., He, B. Y., Li, G., Leung, K. S., et al. (2008). Icaritin, a potential estrogen receptor beta antagonist molecule Icaritin, promote osteoporotic fracture repair in ovariectomized mice: preliminary finding at
3 weeks post fracture. Bone 43 (Suppl. S1), S76-S77. doi:10.1016/j.bone.2008.07. 081

Zhang, G., Qin, L., Sheng, H., Wang, X. L., Wang, Y. X., and Yeung, D. K. (2009). A novel semisynthesized small molecule icaritin reduces incidence of steroidassociated osteonecrosis with inhibition of both thrombosis and lipiddeposition in a dose-dependent manner. Bone 44 (2), 345-356. doi:10.1016/ j.bone.2008.10.035

Zhang, J., Luo, X., Barbieri, D., Barradas, A. M., de Bruijn, J. D., and van Blitterswijk, C. A. (2014). The size of surface microstructures as an osteogenic factor in calcium phosphate ceramics. Acta Biomater. 10 (7), 3254-3263. doi:10.1016/j.actbio.2014.03.021

Zhang, J., Barbieri, D., ten Hoopen, H., de Bruijn, J. D., van Blitterswijk, C. A., and Yuan, H. (2015). Microporous calcium phosphate ceramics driving osteogenesis through surface architecture. J. Biomed. Mater. Res. 103 (3), 1188-1199. doi:10.1002/jbm.a.35272

Conflict of Interest: The authors declare that the research was conducted in the absence of any commercial or financial relationships that could be construed as a potential conflict of interest.

Copyright (c) 2020 Peng, Li, Xu and Lv. This is an open-access article distributed under the terms of the Creative Commons Attribution License (CC BY). The use, distribution or reproduction in other forums is permitted, provided the original author(s) and the copyright owner(s) are credited and that the original publication in this journal is cited, in accordance with accepted academic practice. No use, distribution or reproduction is permitted which does not comply with these terms. 